- Case Report

\title{
A Rare Case of Renal Impairment Caused by Primary Hypothyroidism
}

\author{
Joleen Choy', Julian Yaxley ${ }^{2, *}$, William Yaxley ${ }^{1}$ \\ 'Department of Medicine, Royal Brisbane and Women's Hospital, Brisbane, QLD, Australia \\ ${ }^{2}$ Department of Nephrology, Sunshine Coast University Hospital, Griffith University School of Medicine, Birtinya, QLD, Australia
}

\begin{abstract}
An association between hypothyroidism and renal impairment has rarely been reported in the literature. We describe a case of hypothyroidism that was associated with otherwise unexplained acute kidney impairment, which was reversed with treatment. A 21-year-old female patient presented to her family physician with myalgia, and preliminary investigations revealed an elevated level of creatine kinase and poor renal function. Primary hypothyroidism was diagnosed and no other apparent etiology for renal failure could be identified despite extensive investigations by the Nephrology Department. Notably, the patient's renal impairment showed prompt resolution following thyroid hormone replacement.
\end{abstract}

Keywords: Hypothyroidism; Renal Insufficiency; Hashimoto Disease

Received: August 28, 2017, Revised: September 16, 2017, Accepted: October 12, 2017

*Corresponding Author: Julian Yaxley https://orcid.org/0000-0002-0587-276X

Tel: +61-07-53906000, Fax: +61-07-52020522, E-mail: julianyaxley@yahoo.com.au 


\section{INTRODUCTION}

Thyroid hormones are necessary to maintain the healthy function of multiple organ systems. Hypothyroidism is an important endocrine disorder that can affect renal physiology. ${ }^{1)}$ An association between hypothyroidism and both acute and chronic renal impairment has been recorded previously in the literature; however, this relationship remains an underappreciated medical entity. ${ }^{2,3)}$ We report a case of primary hypothyroidism accompanied by otherwise unexplained acute renal failure in a patient with previously normal renal function. Of note, the patient's renal impairment showed significant correction following thyroid replacement therapy.

\section{CASE REPORT}

A 21-year-old woman was referred to the Internal Medicine Clinic by her family physician with a 4-week history of gradually progressive myalgia, lethargy, and declining renal function. She reported no history of rash, fever, dyspnea, or hematuria. She resided in a metropolitan area of Australia and denied any recent travel or exposure to animals or infectious contacts.

Her medical history was significant for mild childhood asthma and pregnancy 6 months prior to presentation that was complicated by pre-eclampsia for which she underwent a cesarean section at 33 weeks' gestation. The only medication she used was a progesterone contraceptive implant. Her mother had a known history of idiopathic focal segmental glomerulosclerosis, which was being treated with daily administration of mycophenolate and prednisone. Her mother also had history of pulmonary sarcoidosis and was being investigated for newly diagnosed anti-neutrophil cytoplasmic antibody-positive vasculitis.

Upon examination, the patient showed periorbital and bilateral lower limb edema, as well as central obesity. No tonsillitis, painful goiter, muscle tenderness, and/or rash were observed. Her abdomen was

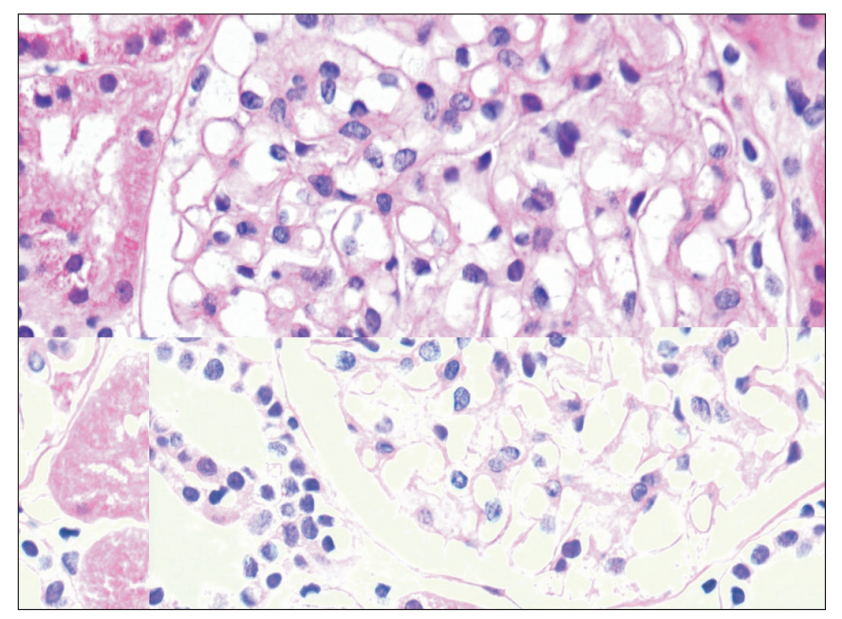

Figure 1. Light microscopy showing normal glomerular morphology with no evidence of tubulointerstitial disease or arteritis (periodic acid-Schiff staining, $\times 400$ ). nontender, and no palpable organomegaly was observed.

Blood tests ordered by her family physician included a complete blood count and assessment of inflammatory biomarkers, which were observed to be within reference range. Her creatine kinase (CK) was markedly elevated at 1,460 U/L (reference range, 30-150 U/L), serum creatinine was $136 \mu \mathrm{mol} / \mathrm{L}$ (reference range, $45-85 \mu \mathrm{mol} / \mathrm{L}$ ), serum urea $4.9 \mathrm{mmol} / \mathrm{L}$ (reference range, $2.5-6.5 \mathrm{mmol} / \mathrm{L}$ ), and her glomerular filtration rate (GFR) was $43 \mathrm{~mL} / \mathrm{min} / 1.73 \mathrm{~m}^{2}$ (reference range, > 59 $\mathrm{mL} / \mathrm{min} / 1.73 \mathrm{~m}^{2}$ ). Baseline blood testing performed during antenatal care 6 months prior had revealed a normal GFR of $90 \mathrm{~mL} / \mathrm{min} / 1.73$ $\mathrm{m}^{2}$, creatinine $77 \mu \mathrm{mol} / \mathrm{L}$, and urea $3.0 \mathrm{mmol} / \mathrm{L}$. Local laboratories measure creatinine using the alkaline picrate method, and calculate GFR using the Modification of Diet in Renal Disease Study equation. ${ }^{1)}$ Autoimmune markers and viral serology were negative.

Results of urine microscopy and culture were normal. No microalbuminuria or proteinuria were identified; however, urinary myoglobin was elevated $(99 \mu \mathrm{g} / \mathrm{L}$; reference range, $<70 \mu \mathrm{g} / \mathrm{L})$. Ultrasonography of the urinary tract did not reveal any remarkable findings.

Thyroid function tests (TFTs) were performed at the Internal Medicine Clinic to investigate the elevated CK-these confirmed a diagnosis of primary hypothyroidism. Her free thyroxine (T4) level was 3.2 $\mathrm{pmol} / \mathrm{L}$ (reference range, 7-17 pmol/L), and her thyroid stimulating hormone (TSH) level was $160 \mathrm{mU} / \mathrm{L}$ (reference range, 0.3-4.5 mU/L). Thyroid autoantibodies were positive. Thyroxine at a dose of $100 \mathrm{mcg}$ daily was initiated to treat hypothyroidism, and she was referred to the Department of Nephrology for further evaluation of her renal impairment. Renal biopsy was performed that same week and demonstrated no histopathological abnormalities (Figures 1,2).

Following initiation of thyroxine, the patient's myalgia and lethargy showed complete resolution at her 2-week follow-up with her family physician. CK had reduced to $462 \mathrm{U} / \mathrm{L}$ and GFR had normalized to 80 $\mathrm{mL} / \mathrm{min} / 1.73 \mathrm{~m}^{2}$. Her TFTs had improved to a T4 level of $7.8 \mathrm{pmol} / \mathrm{L}$, and TSH was trending downwards at $71 \mathrm{mU} / \mathrm{L}$. At the 3-month outpa-

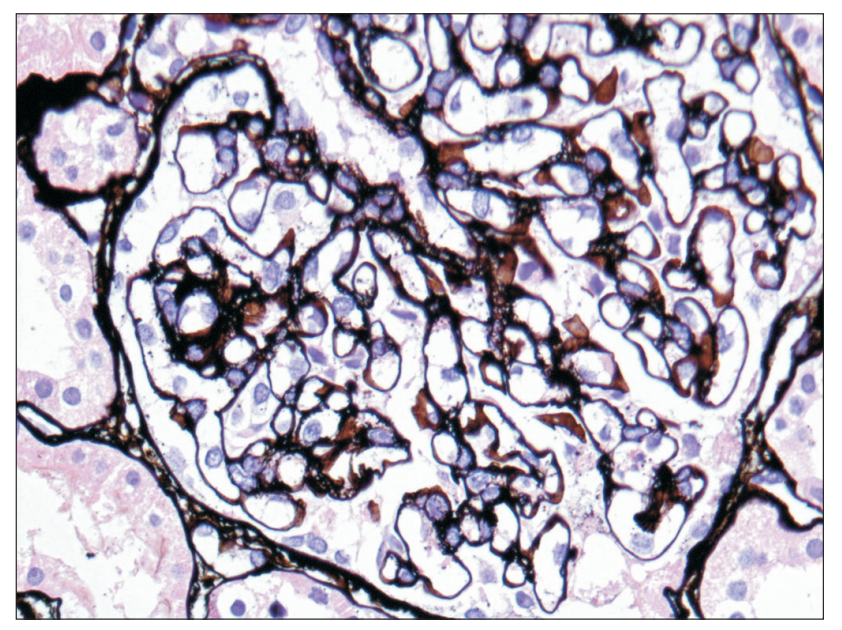

Figure 2. Normal silver staining of the capillary basement membrane (silver methenamine stain, $\times 400$ ). 
tient follow-up, the patient remained stable with the use of thyroxine and showed normal renal function.

\section{DISCUSSION}

Hypothyroidism is thyroid gland underactivity associated with inadequate production of thyroid hormones. The prevalence of hypothyroidism is approximately 10 -fold higher in women than in men. ${ }^{2)}$ The most common etiological factor associated with this condition globally is iodine deficiency; however, in countries with a sufficient iodine intake observed in the population, Hashimoto's thyroiditis is the most common cause. ${ }^{3)}$ Both Hashimoto's and postpartum thyroiditis are autoimmune-mediated processes associated with the development of positive thyroid autoantibodies, which cause hypothyroidism. Whether hypothyroidism resulted from Hashimoto's thyroiditis or postpartum thyroiditis in our patient (as opposed to case), remains unclear.

Thyroid hormones play a fundamental role in the functioning of multiple organ systems. They are involved in the regulation of metabolism, body temperature, protein synthesis, growth and development, hemodynamics, and water and electrolyte homeostasis. Clinical features of hypothyroidism may be subtle. Hypothyroidism, both overt and subclinical, can be associated with elevated CK levels and derangement in liver biochemistry suggesting abnormal skeletal muscle and hepatic metabolism. ${ }^{4)}$

This patient presented with subtle signs of hypothyroidism and acute renal impairment. We propose that this phenomenon is directly attributable to primary hypothyroidism. The patient's low GFR showed rapid and near-complete reversal following the administration of thyroxine. No other pre- or post-renal or intrinsic renal processes were identified as likely etiological contributors to her kidney dysfunction. Her markedly elevated CK was consistent with a myopathy associated with hypothyroidism and also improved after the initiation of treatment.

Multiple factors have been suspected to play a role in the pathogenesis of kidney failure secondary to hypothyroidism. These include decreased kidney mass, reduced renal perfusion pressure because of decreased cardiac output and increased vascular resistance, reduced sensitivity to the body's sympathetic drive and renin-angiotensin-aldosterone system (RAAS) activity, as well as possible rhabdomyolysis. Thyroid hormone replacement increases the functioning renal mass, improves cardiac output, enhances catecholamine responsiveness and RAAS activity, and reverses rhabdomyolysis. The GFR is reversibly reduced by nearly $40 \%$ in approximately $55 \%$ of hypothyroid adults, and thyroid hormone replacement usually improves or resolves their renal impairment. ${ }^{5)}$

Renal biopsies performed in hypothyroid patients classically demonstrate thickening of the glomerular and tubular basement membranes and cytoplasmic inclusions. ${ }^{5)}$ Histopathological reversibility following thyroid hormone replacement is variable among patients. ${ }^{6)}$ A renal biopsy performed in our patient did not demonstrate any abnormal features suggesting hypothyroid-mediated changes.

TFTs constitute simple and non-invasive blood tests that should be incorporated into the basic work-up of patients presenting with unexplained derangement of renal function. These tests can be easily performed in primary care settings, and our case demonstrates that invasive investigations like renal biopsies can be avoided. The association between hypothyroidism and renal failure has been under-recognized. A greater acknowledgment of this relationship by treating clinicians could greatly benefit patients.

\section{CONFLICT OF INTEREST}

No potential conflict of interest relevant to this article was reported.

\section{REFERENCES}

1. Levey AS, Bosch JP, Lewis JB, Greene T, Rogers N, Roth D. A more accurate method to estimate glomerular filtration rate from serum creatinine: a new prediction equation. Modification of Diet in Renal Disease Study Group. Ann Intern Med 1999;130:461-70.

2. Vanderpump MP. The epidemiology of thyroid disease. Br Med Bull 2011;99:39-51.

3. Garber JR, Cobin RH, Gharib H, Hennessey JV, Klein I, Mechanick JI, et al. Clinical practice guidelines for hypothyroidism in adults: cosponsored by the American Association of Clinical Endocrinologists and the American Thyroid Association. Endocr Pract 2012;18:9881028.

4. Beyer IW, Karmali R, Demeester-Mirkine N, Cogan E, Fuss MJ. Serum creatine kinase levels in overt and subclinical hypothyroidism. Thyroid 1998;8:1029-31.

5. Montenegro J, Gonzalez O, Saracho R, Aguirre R, Gonzalez O, Martinez I. Changes in renal function in primary hypothyroidism. Am J Kidney Dis 1996;27:195-8.

6. Fauci AS, Kasper DL, Longo DL, Braunwald E, Hauser SL, Jameson JL, et al. Harrison's principles of internal medicine. 17th ed. New York (NY): McGraw-Hill Medical; 2008. 